\title{
Opioid-induced constipation in patients with chronic noncancer pain in the USA, Canada, Germany, and the UK: descriptive analysis of baseline patient-reported outcomes and retrospective chart review
}

This article was published in the following Dove Press journal:

ClinicoEconomics and Outcomes Research

23 May 2014

Number of times this article has been viewed

\section{Karin S Coyne' \\ Robert J LoCasale ${ }^{2}$ \\ Catherine J Datto ${ }^{2}$ \\ Chris C Sexton' \\ Karen Yeomans ${ }^{3}$ \\ Jan Tack ${ }^{4}$}

'Evidera, Bethesda, MD, ${ }^{2}$ AstraZeneca, Wilmington, DE, USA; ${ }^{3} \mathrm{UBC}$ : an Express Scripts Company, Montreal, QC, Canada; ${ }^{4}$ University Hospital Gasthuisberg, Leuven, Belgium
Correspondence: Karin S Coyne Evidera, 710I Wisconsin Ave, Suite 600, Bethesda, MD 208I4, USA

$\mathrm{Tel}+\mathrm{I} 30 \mathrm{I} 6549729$

Fax +I 30I 6549864

Email karin.coyne@evidera.com
Background: The characteristics of patients who suffer from noncancer pain and opioid-induced constipation are not well understood.

Methods: Cross-sectional patient survey and chart review data from the baseline assessment of an ongoing longitudinal study in the USA, Canada, Germany, and the UK were evaluated via descriptive statistics. Participants had confirmation of daily opioid therapy $\geq 30 \mathrm{mg}$ for $\geq 4$ weeks and self-reported opioid-induced constipation. Response to laxatives was defined by classifying participants into categories of laxative use and evaluating the prevalence of inadequate response to one laxative agent and two or more agents from at least two different laxative classes. Outcomes included the Patient Assessment of Constipation-Symptoms, Work Productivity and Activity Impairment Questionnaire-Specific Health Problem, EuroQOL 5 Dimensions, and Global Assessment of Treatment Benefit, Satisfaction, and Willingness to Continue.

Results: Patients reported a mean of 1.4 bowel movements not preceded by laxatives and 3.7 bowel movements with laxative use per week; $83 \%$ wanted at least one bowel movement per day. Most commonly reported on Patient Assessment of Constipation-Symptoms were straining/squeezing to pass bowel movements (83\%), bowel movements too hard $(75 \%)$, flatulence (69\%), and bloating (69\%). Eighty-four percent were taking natural or behavioral therapies; $60 \%$ were taking at least one over-the-counter laxative; and 19\% were taking at least one prescription laxative. Prevalence of inadequate response to one laxative agent was $94 \%$; inadequate response to two or more agents from at least two different laxative classes was $27 \%$. Mean Work Productivity and Activity Impairment Questionnaire-Specific Health Problem values for percent work time missed, percent impairment while working, and percent activity impairment were $9 \%, 32 \%$ (equivalent of 14 hours of lost productivity per week), and 38\%. Mean EuroQOL 5 Dimensions index and visual analog scale scores were 0.49 and 50.6, respectively. Forty-four percent reported being satisfied with their treatment for constipation.

Conclusion: Patients treated with opioids for noncancer pain commonly endure constipation symptoms that limit their work productivity and overall health-related quality of life while adhering to treatments that provide little relief. Further research is needed to identify more efficacious constipation therapies for this patient population.

Keywords: opioid, constipation, pain, laxatives, quality of life 


\section{Introduction}

Opioid analgesics are increasingly being used for the treatment of chronic noncancer pain. ${ }^{1}$ Despite proven analgesic efficacy, the location of $\mu$-opioid receptors in the gastrointestinal tract is associated with dose-limiting constipation that seriously impacts patients' health-related quality of life. Symptoms include difficult and infrequent passage of hard stools, often accompanied by straining and incomplete evacuation. ${ }^{2}$ Opioid-induced constipation (OIC) has been shown to impair patients' abilities to carry out their activities of daily living and to result in greater work time missed and diminished work productivity, as well as lower levels of overall health and well-being. ${ }^{3-6}$

Estimates of the prevalence of OIC vary according to study design and patient population, ranging from $15 \%$ to $90 \%$ based on an analysis of 16 clinical trials and observational studies identified in a recent systematic review. ${ }^{7,8}$ When qualified according to type of chronic pain, estimates from observational studies in the USA suggest that the prevalence of OIC in patients with noncancer pain ranges between $40 \%$ and $50 \%{ }^{4,8-11}$

Agents commonly used to manage OIC, such as laxatives and bicyclic fatty acids, do not address the full spectrum of underlying opioid receptor-mediated causes of constipation, may cause considerable gastrointestinal side effects, and are often ineffective. ${ }^{12}$ A Cochrane systematic review conducted in palliative care patients evaluated the efficacy of laxatives in seven studies involving 616 people. ${ }^{13}$ The drugs evaluated were lactulose, senna, danthron combined with poloxamer, misrakasneham, magnesium hydroxide combined with liquid paraffin, and methylnaltrexone. While initial findings showed that methylnaltrexone improved bowel relaxation as compared with placebo, there were no clear treatment benefits for any of the other drugs, in part due to the multiplicity of use of different agents. In parallel, data from the US and European PROBE I (Prospective Randomized Open Blinded End-point) survey of patients who reported taking daily oral opioids and laxatives $(\mathrm{n}=322)$ found that $81 \%$ had constipation and $45 \%$ had less than three spontaneous bowel movements per week. ${ }^{4}$ One third (33\%) of patients had missed, decreased, or even stopped using opioids specifically in order to make it easier to have a bowel movement, which resulted in increased pain in $92 \%$ of cases. ${ }^{4}$

Despite the cited references, the characteristics of patients who suffer from noncancer pain and OIC in clinical practice have not been fully elucidated. Data on laxative utilization patterns and the effectiveness and tolerability of therapies for constipation in this patient population are sparse. Therefore, a hybrid, prospective longitudinal study is being conducted to generate real-world empirical evidence to better characterize patients with noncancer pain who develop constipation. The present study is an analysis of the completed baseline data that aims to characterize demographics, clinical characteristics, constipation symptoms, pain management, therapies used for the treatment of OIC, rate of inadequate response to laxatives, and the impact of OIC on work productivity in a multicountry sample of patients with chronic noncancer pain and OIC.

\section{Materials and methods}

\section{Study design and sample}

This longitudinal study is ongoing in the USA, Canada, Germany, and the UK to assess the burden of OIC in patients with noncancer pain using a combination of a patient survey, retrospective data abstraction from medical records, and physician survey. The study received institutional review board approval and was executed in accordance with the Canadian regulation known as the Personal Information Protection and Electronic Documents Act, the Health Insurance Portability and Accountability Act of 1996, the European Union Data Protection Directive, and the Safe Harbour agreement. The protocol (NCT01928953) was also reviewed and endorsed by the Anaesthetics Pain Subgroup and the Primary Care Research Network. The patient-reported component includes one Internet-based baseline survey and eight follow-up Internet surveys over 24 weeks (weeks 2, 4, 6, 8, 12, 16, 20, and 24). The retrospective chart review is completed via the Internet at baseline and again at the end of the study period (week 24) for each patient who completed a baseline survey. The Internet-based patient-specific physician survey is completed at baseline and at week 24 for each patient who completed a baseline web-based survey. This manuscript presents the analysis of the completed baseline data from the patientreported survey and retrospective chart review. Details about the longitudinal study and data will be described in a separate paper following study completion.

The study sample comprised patients recruited from primary care clinics, pain management clinics, and clinical research sites affiliated with primary care networks who had chart review confirmation of daily opioid therapy lasting $\geq 4$ weeks for the treatment of chronic noncancer pain and who reported OIC during their screening interview. A cut point of $\geq 4$ weeks was selected to ensure patients were consistently treated with opioids (eg, not for acute injury or post-surgical use). The minimum daily opioid dose for study inclusion was $30 \mathrm{mg}$ of oral morphine or equianalgesic 
amount(s) of one or more other opioid therapies. Interested participants from this target population who elected to complete the baseline survey were further evaluated for the presence of OIC based on their responses, to confirm study eligibility (Table 1).

Participant recruitment was stratified with the goal of recruiting patients from two subgroups based on duration of exposure to opioid therapy: ${ }^{14}$ those with $\geq 4$ weeks but $\leq 2$ years of routine opioid use and those with $>2$ years of routine opioid use.

\section{Data collection}

Clinical site staff identified a preliminary cohort of potentially eligible patients by review of the medical charts at their site. Once identified, staff then described the study and the informed consent process to each patient. Interested and eligible patients were provided with a welcome email and a welcome letter, including instructions as to how to access the online survey enrollment module using a unique uniform resource locator with an embedded password. Patient information was recorded in a patient tracking sheet, with only the sites having access to patient identifying information in order to retrieve medical records for data abstraction during the retrospective chart review. Following completion of the baseline survey, participants were compensated for their time in the form of an electronic gift card in the country's currency, with values ranging from the equivalent of 15 to 25 USD.

The retrospective chart review was performed by the site staff via an Internet-based platform designed to capture patient data related to medical history, pharmacotherapy (including opioid and laxative prescriptions), and health care resource utilization. Lastly, physicians completed an Internet-based questionnaire regarding their perception of the patient's OIC burden, symptoms, treatment patterns for OIC, laxative use, and overall OIC treatment satisfaction, the data for which are forthcoming. Additional information about the content of each of the sources of data collection analyzed in the present study is provided below.

\section{Patient questionnaire}

The patient-reported questionnaire included questions from standardized questionnaires supported by evidence of reliability and validity as well as questions developed for this study. The standardized questionnaires were as follows.

\section{Patient Assessment of Constipation-Symptoms}

The Patient Assessment of Constipation-Symptoms questionnaire (PAC-SYM) ${ }^{15}$ was designed to assess patient-reported
Table I Study eligibility criteria and definitions of laxative sufficiency and inadequate response

OIC (criteria for - Participants who respond "yes" to the confirmation of study eligibility) baseline question: "Since starting the opioid pain medication, have you been experiencing constipation or worsening of current constipation? Yes/No") OR

- Patient must report laxative use (see definition below) if the number of BMs in the past two weeks is $\geq 3$ per week OR

- Patients with less than three BMs per week in the past 2 weeks were eligible, regardless of whether they report laxative use OR

- Patients who did not report laxative use but reported at least one symptom of OIC at least "moderate" or more often on the PAC-SYM screening questions in the past 2 weeks (ie, incomplete BM, BM too hard, straining during $B M$, or sensation of false alarm)

Laxative classes $^{\mathrm{a}}$

- Stool softeners, eg, docusate sodium (Colace ${ }^{\circledR}$, Ducolax ${ }^{\circledR}$ stool softener), mineral oil

- Osmotics, eg, polyethylene glycol 3350 (Miralax ${ }^{\circledast}$, Dulcolax ${ }^{\circledR}$ balance)

- Stimulants, eg, bisacodyl (Dulcolax ${ }^{\circledR}$ laxative), senna $\left(\right.$ Ex-Lax $^{\circledR}$, Senokot $\left.^{\circledR}\right)$

- Salines, eg, magnesium hydrochloride (milk of magnesia, Citroma ${ }^{\circledR}$ )

- Rectal options (suppositories, enemas)

- Prescription laxative treatments, eg, polyethylene glycol 3350, lactulose (Constulose ${ }^{\circledR}$ ), lubiprostone (Amitiza ${ }^{\circledR}$ ), methylnaltrexone bromide (Relistor ${ }^{\circledR}$ )

Sufficient laxative use Use of at least one laxative $\geq 4$ times over the last 2 weeks

Insufficient laxative use Use of laxatives less than four times but $\geq I$ time over the last 2 weeks

Nonlaxative use IX LIR

No reported laxative use over the last 2 weeks Sufficient laxative use (use of at least one laxative agent from a class $\geq 4$ times in the last 2 weeks) and inadequate response, defined as $<3$ BMs AND $\geq$ I PAC-SYM symptom scored moderate, severe, or very severe

$2 \times \operatorname{LIR}$ Sufficient laxative use of agents from two different classes (use of at least two laxative agents from at least two different classes $\geq 4$ times each in the last 2 weeks) and inadequate response, defined as $<3$ BMs AND $\geq$ I PAC-SYM symptom scored moderate, severe, or very severe

Notes: aparticipants were asked about their use of a range of behavioral interventions and therapeutic agents via a series of pictures with brand name and generic drug information provided. In order to provide a more stringent definition of inadequate response, the following classes were considered laxatives: stool softeners, stimulants, saline, rectal, and prescription laxative treatments. Probiotics, natural diet changes, increased fluids, increased exercise/activity, and OTC fiber supplements were not defined as laxatives for the purposes of defining inadequate response. However, information about utilization of these treatments is summarized in Table 5.

Abbreviations: BM, bowel movement; LIR, laxative-inadequate responders; I $\times$ LIR, inadequate response to one laxative agent; $2 \times \operatorname{LIR}$, inadequate response to $\geq 2$ agents from $\geq 2$ different laxative classes; OIC, opioid-induced constipation; OTC, over-thecounter; PAC-SYM, Patient Assessment of Constipation-Symptoms. 
symptoms and the severity of symptoms over the previous 2 weeks. The 12-item measure assesses three subscales, ie, stool, rectal, and abdominal symptoms. Each item is rated on a five-point Likert scale, ranging from 0 to 4, where a higher score indicates greater symptom severity. The PAC-SYM has been widely used in OIC medication treatment studies, and has performed as expected in relation to clinical outcomes. ${ }^{16-20}$

\section{Work Productivity and Activity Impairment Questionnaire, Specific Health Problem}

The Work Productivity and Activity Impairment QuestionnaireSpecific Health Problem (WPAI-SHP, version 2.0) is a selfreported quantitative assessment of the effect of specific health problems, in this case, constipation, on work productivity, daily activities, and classroom impairment over the past 7 days. ${ }^{21}$ WPAI-Constipation outcomes are expressed as impairment percentages, with higher scores indicating greater impairment and less productivity, ie, worse outcomes. Outcomes include the following: percent work time missed due to constipation (absenteeism); percent impairment while working due to constipation (presenteeism); percent impairment in overall work productivity based on responses to questions about time missed due to constipation; and percent daily activity impairment (impairment in activities other than work due to constipation). This tool has been adapted for use and supported by evidence of reliability, validity, and responsiveness across a range of therapeutic areas, including irritable bowel syndrome (IBS) with chronic constipation. ${ }^{22-24}$

\section{EuroQOL 5 Dimensions}

The EuroQOL 5 Dimensions (EQ-5D) is a five-item health state descriptive system where full health is one, and zero is equivalent to death (EQ-5D Index) and a visual analog scale (EQ-5D VAS, range 0-100 from worst to best imaginable health state). There is extensive evidence supporting the reliability and validity of the EQ-5D, ${ }^{25}$ and it has performed well in a range of therapeutic areas, including OIC, ${ }^{5} \mathrm{IBS},{ }^{26}$ and chronic pain. ${ }^{27,28}$ The EQ-5D was completed by patients on site in a paper-based format and the data were entered into the Internet-based data capture system. Scoring was based on developer guidelines and weighted by country. ${ }^{25}$

\section{Global Assessment of Treatment Benefit, Satisfaction, and Willingness to Continue}

The Global Assessment of Treatment Benefit, Satisfaction, and Willingness to Continue (BSW) consists of three singleitem measures that are designed to capture patients' perception of the effect of their treatment in terms of benefits, satisfaction, and willingness to continue treatment. ${ }^{29}$ The BSW has demonstrated evidence of construct validity in patients with overactive bladder and has been used in urology treatment studies. ${ }^{29,30}$

Other questions were developed specifically for the patient survey to assess demographic and clinical characteristics, treatment patterns, frequency of bowel movements, additional constipation symptoms not evaluated on the PAC-SYM, pain management, and utilization of therapies for the treatment of OIC.

\section{Chart review questionnaire}

Outcomes from the chart review that was available for evaluation in this baseline analysis included questions about clinical history, prescribed pharmacotherapy, and comorbid conditions.

\section{Statistical analysis}

All analyses were performed using SAS version 9.2 (SAS Institute, Cary, NC, USA) following a statistical analysis plan approved prior to receipt of the locked and clean dataset. The electronic patient surveys included preprogrammed validation checks and error messages to help guide patients through the surveys, while controlling data quality and integrity at the point of data entry. Data from the chart review were checked for completeness by the research team, and sites were asked to complete missing responses to the extent possible. The data were analyzed as observed, without imputation for missing responses.

Participants from the target population who completed the baseline survey were further evaluated for presence of OIC in order to confirm study eligibility (Table 1). Descriptive statistics were used to evaluate outcomes for the overall sample and by country. For continuous variables, the mean and standard deviation was described and rounded to one decimal for all data. For categorical variables, the number and percent distribution by category was described. Participants' reports of treatments for constipation were classified as: natural/behavior therapies, which include probiotics, natural dietary changes, increased fluids, increased exercise, and fiber supplements; over-the-counter laxatives, which include stool softeners, osmotic laxatives, saline laxatives, rectal options, and other over-the-counter laxatives; and prescription laxatives, including osmotic laxatives, lactulose, lubiprostone, methylnaltrexone, and other prescription agents. Response to laxatives was defined by first classifying participants into three categories: sufficient (at least 4 days per week) laxative use, insufficient laxative use, 
and nonlaxative use, and then evaluating the prevalence of inadequate response to one laxative agent and to at least two or more agents from at least two different laxative classes (Table 1). These categories were created to quantify laxative exposure in relation to other outcomes and do not imply clinical sufficiency.

\section{Results}

A total of 617 patients were recruited. Of these, 500 patients ( $81 \%$ ) completed the baseline patient questionnaire, and 493 (99\%) met criteria confirming the presence of OIC (USA, 242; Canada, 38; Germany, 115; UK, 98). Sixty-two percent of participants were female, and most $(85 \%)$ were Caucasian. The mean ( \pm standard deviation) age was $52.6 \pm 11.6$ years. About $60 \%$ were married, living with a partner, or in a common law partnership, and less than a third (27\%) were working full-time or part-time. Thirty-five percent reported that they were unable to work due to disability.

Sixty-three percent of patients reported discussing their constipation symptoms resulting from their opioid medication use during an office visit with their health care provider (Table 2). Among patients who did not discuss their constipation problems with their health care provider, commonly reported reasons included having discussed such problems with the health care provider in the past (59\%), being concerned that they would need to change/reduce pain medication (14\%), and being embarrassed (9\%). Chronic back and joint pain were the most frequently reported conditions (77\% and $52 \%$, respectively). Mean duration of chronic pain and opioid use were 9.8 \pm 8.9 (median 7.0) and 6.4 6 6.3 (median 4.3) years, respectively. Recruiting patients with less than 2 years of opioid medication use proved difficult; the final sample was composed of $24 \%$ who reported $<2$ years of opioid medication use and $65 \%$ who reported $\geq 2$ years, with the remaining patients reporting that they were unsure of the duration of opioid use. Participants commonly reported that their constipation interfered with the ability of their opioid medication to control pain, with $49 \%$ reporting moderate or complete interference and $8 \%$ reporting that they changed how they used their opioid in order to have a bowel movement (Table 2).

Data from the 482 patients with a completed chart review showed that $97 \%$ were taking more than one class of chronic pain medication. In addition to opioids, anticonvulsants $(23 \%)$, nonsteroidal anti-inflammatories (17\%), muscle relaxants $(14 \%)$, and antidepressants $(11 \%)$ were frequently coprescribed for pain. The most common opioid prescribed was oxycodone $(42 \%)$, followed by morphine
$(16 \%)$, hydrocodone $(16 \%)$, and tramadol (15\%, Table 3$)$. Over half of the participants had documentation of at least one of the prespecified comorbid conditions assessed on the chart review form $(61 \%)$. The most common concomitant medications prescribed for reasons other than chronic pain were antidepressants (27\%), anticonvulsants (19\%), antacids (18\%), and antihypertensive medications $(17 \%$, Table 3).

Symptom burden was prevalent within the study population. Patients reported a mean of $1.4 \pm 2.3$ spontaneous bowel movements not preceded by laxatives per week and a mean of $3.7 \pm 2.9$ bowel movements overall per week; however, $83 \%(n=407)$ reported wanting to have a bowel movement at least once a day or more often. The most commonly reported symptoms of moderate or greater severity on the PAC-SYM were straining/squeezing to pass a bowel movement (83\%), bowel movements that were too hard (75\%), flatulence (69\%), an incomplete bowel movement (68\%), bloating in the abdomen (69\%), painful bowel movements (67\%), and abdominal discomfort (64\%, Table 4).

The most frequently utilized OIC treatments were natural or behavioral therapies (84\%). Sixty percent used at least one over-the-counter laxative, $24 \%$ used two or more over-thecounter laxatives, and $19 \%$ used one or more prescription laxatives (Table 5). Over one third (36\%) of patients reported no laxative use at all in the past 2 weeks, another $24 \%$ were considered to have insufficient laxative use and $40 \%$ to have sufficient laxative use based on prespecified assessments (Table 5; see Table 1 for definitions of classifications). The rate of inadequate response to at least one laxative agent within the past 2 weeks was 94\%. Inadequate response to use of at least two laxative agents from two or more different classes at least four times each within the past 2 weeks was 27\% (Table 6).

Patient-reported outcomes on WPAI-Constipation, the EQ-5D, and BSW reflected the considerable impact of OIC on daily functioning and suggest that the treatments patients utilized for OIC had moderate benefits associated with modest levels of overall satisfaction (Tables 7 and 8). Just over one quarter (27\%) of participants in this chronic pain sample were currently employed (Table 7). Participants who were employed reported missing a mean of $4.6 \pm 11.9$ hours of work over the previous 7 days because of problems associated with their constipation, with the greatest mean hours missed reported in the USA (6.2 \pm 13.9$)$. Mean WPAI-Constipation values for percent work time missed and percent impairment while working due to constipation were $9 \%$ and $32 \%$, respectively (Table 7 ). When all participants 
Table 2 Patient-reported clinical characteristics and pain

\begin{tabular}{|c|c|c|c|c|c|}
\hline & $\begin{array}{l}\text { Overall } \\
(n=493)\end{array}$ & $\begin{array}{l}\text { USA } \\
(n=242)\end{array}$ & $\begin{array}{l}\text { Canada } \\
(n=38)\end{array}$ & $\begin{array}{l}\text { Germany } \\
(n=\mid 15)\end{array}$ & $\begin{array}{l}\text { UK } \\
(n=98)\end{array}$ \\
\hline \multicolumn{6}{|l|}{ Discussed OIC with HCP } \\
\hline Yes, n (\%) & $310(62.9)$ & $156(64.5)$ & $23(60.5)$ & $87(75.7)$ & $44(44.9)$ \\
\hline \multicolumn{6}{|l|}{ Health rating, n (\%) } \\
\hline Excellent & $7(1.4)$ & $5(2.1)$ & I (2.6) & I (0.9) & $0(0.0)$ \\
\hline Very good & $33(6.7)$ & $23(9.5)$ & $4(10.5)$ & $2(1.7)$ & $4(4.1)$ \\
\hline Good & $147(29.8)$ & $97(40.1)$ & $13(34.2)$ & $12(10.4)$ & $25(25.5)$ \\
\hline Fair & $207(42.0)$ & $91(37.6)$ & $16(42.1)$ & $66(57.4)$ & $34(34.7)$ \\
\hline Poor & $96(19.5)$ & $24(9.9)$ & $4(10.5)$ & $33(28.7)$ & $35(35.7)$ \\
\hline Missing & $3(0.6)$ & $2(0.8)$ & $0(0.0)$ & I (0.9) & $0(0.0)$ \\
\hline \multicolumn{6}{|l|}{ Type of chronic pain, $n(\%)^{\mathrm{a}}$} \\
\hline Back pain & $379(76.9)$ & $209(86.4)$ & $23(60.5)$ & 79 (68.7) & $68(69.4)$ \\
\hline Joint pain & $256(51.9)$ & $126(52.1)$ & $17(44.7)$ & $58(50.4)$ & $55(56.1)$ \\
\hline Fibromyalgia & $74(15.0)$ & $29(12.0)$ & $14(36.8)$ & $20(17.4)$ & II (II.2) \\
\hline Headache or migraine & $80(16.2)$ & $48(19.8)$ & $5(13.2)$ & $15(13.0)$ & $12(12.2)$ \\
\hline Osteoarthritis & $95(19.3)$ & $47(19.4)$ & II (28.9) & $5(4.3)$ & $32(32.7)$ \\
\hline Rheumatoid arthritis & $4 \mid(8.3)$ & $24(9.9)$ & $3(7.9)$ & $9(7.8)$ & $5(5.1)$ \\
\hline Neuralgia & $115(23.3)$ & $48(19.8)$ & $14(36.8)$ & $31(27.0)$ & $22(22.4)$ \\
\hline Pain syndrome & $163(33.1)$ & $21(8.7)$ & $6(15.8)$ & $83(72.2)$ & $53(54.1)$ \\
\hline Other & $8(1.6)$ & $3(1.2)$ & $0(0.0)$ & $5(4.3)$ & $0(0.0)$ \\
\hline \multicolumn{6}{|l|}{ Pain severity } \\
\hline Mean pain last 24 hours (SD) & $6.3(1.8)$ & $6.4(1.7)$ & $6.2(1.9)$ & $5.6(1.9)$ & $6.7(1.8)$ \\
\hline Mean pain last 7 days (SD) & $6.3(1.7)$ & $6.4(1.6)$ & $6.3(1.9)$ & $5.7(2.0)$ & $6.9(1.5)$ \\
\hline $\begin{array}{l}\text { How much does the constipation interfere with the } \\
\text { ability of opioid medication to control pain? n (\%) }\end{array}$ & $n=461$ & $\mathrm{n}=235$ & $n=37$ & $\mathrm{n}=98$ & $\mathrm{n}=91$ \\
\hline No interference; pain adequately managed & $89(19.3)$ & $34(14.5)$ & $8(21.6)$ & $27(27.6)$ & $20(22.0)$ \\
\hline Little interference; pain mostly managed & $147(31.9)$ & $80(34.0)$ & $14(37.8)$ & $30(30.6)$ & $23(25.3)$ \\
\hline Moderate interference; pain moderately managed & $203(44.0)$ & $115(48.9)$ & $14(37.8)$ & $35(35.7)$ & $39(42.9)$ \\
\hline $\begin{array}{l}\text { Complete interference with adequate pain } \\
\text { management; pain not at all managed }\end{array}$ & $22(4.8)$ & $6(2.6)$ & I (2.7) & $6(6.1)$ & $9(9.9)$ \\
\hline $\begin{array}{l}\text { In the past } 7 \text { days, did you change how you used your opioid } \\
\text { medication(s) so that you could have BMs? (n, \% yes) }\end{array}$ & $37(7.5)$ & $23(9.5)$ & $2(5.3)$ & I (0.9) & II (II.2) \\
\hline If yes, how did you change? ( $n, \%)$ & $n=37$ & $n=23$ & $n=2$ & $n=1$ & $\mathrm{n}=\mathrm{II}$ \\
\hline No longer take my pain medication & $\mathrm{I}(2.7)$ & $0(0.0)$ & $0(0.0)$ & $0(0.0)$ & I (9.I) \\
\hline Reduced how much of my pain medication I use & $16(43.2)$ & II (47.8) & I $(50.0)$ & $0(0.0)$ & $4(36.4)$ \\
\hline Temporarily interrupted the use of pain medication & $18(48.6)$ & $14(60.9)$ & $0(0.0)$ & $0(0.0)$ & $4(36.4)$ \\
\hline Switched to a different pain medication & $4(10.8)$ & I (4.3) & $0(0.0)$ & $\mathrm{I}(100.0)$ & $2(18.2)$ \\
\hline Other & $\mathrm{I}(2.7)$ & $0(0.0)$ & I (50.0) & $0(0.0)$ & $0(0.0)$ \\
\hline
\end{tabular}

Note: ${ }^{\mathrm{T} T h i s ~ c a t e g o r y}$ is not mutually exclusive and participants were able to report more than one type of chronic pain.

Abbreviations: BMs, bowel movements; SD, standard deviation; HCP, health care provider; OIC, opioid-induced constipation.

were asked to rate the impact of their symptoms on their activities regardless of their employment status, the mean percent activity impairment due to constipation was $38 \%$. The mean EQ-5D index score was $0.49 \pm 0.29$, and the mean EQ-5D VAS score was 50.6 \pm 21.6 .

Sixty-seven percent of participants reported on the BSW questionnaire that they had some benefit from their treatment for constipation, with the majority of those who reported benefit characterizing it as "little" (56\%, Table 8). Less than half of participants reported being satisfied with their constipation treatment (44\%). Nonetheless, the overwhelming majority of participants (94\%) reported being willing to continue their current treatment for constipation.

\section{Discussion}

To our knowledge, this is the first observational study to provide real-world data from patients with chronic noncancer pain and OIC with confirmation of opioid use and clinical characteristics from chart review. Results from this analysis of the completed baseline data suggest substantial unmet OIC treatment needs. First, these results confirm that patients with chronic pain and OIC frequently report moderate to severe gastrointestinal symptoms. While the present study used the PAC-SYM questionnaire to evaluate these symptoms, and thus includes item wording and response options that are not directly comparable with some prior studies, the symptoms reported most frequently here are consistent with other 
Table 3 Chart review of clinical characteristics

\begin{tabular}{|c|c|c|c|c|c|}
\hline & Overall $(n=482)$ & USA $(n=237)$ & Canada $(n=35)$ & Germany (n=||4) & UK $(n=96)$ \\
\hline \multicolumn{6}{|l|}{ Chronic pain medications (n, \%) ${ }^{a}$} \\
\hline Alkaloid & $\mathrm{I}(0.2)$ & $0(0.0)$ & $0(0.0)$ & $0(0.0)$ & $\mathrm{I}(\mathrm{I} .0)$ \\
\hline Anesthetic & $15(3.1)$ & $5(2.1)$ & $0(0.0)$ & $3(2.6)$ & $7(7.3)$ \\
\hline Anticonvulsant & $112(23.2)$ & $39(16.5)$ & $9(25.7)$ & $38(33.3)$ & $26(27.1)$ \\
\hline Antidepressant/SNRI & $52(10.8)$ & $10(4.2)$ & $4(\mathrm{II} .4)$ & $20(17.5)$ & $18(18.8)$ \\
\hline Barbiturate & $\mathrm{I}(0.2)$ & I (0.4) & $0(0.0)$ & $0(0.0)$ & $0(0.0)$ \\
\hline Benzodiazepine & $20(4.1)$ & $12(5.1)$ & $4(I I .4)$ & $2(1.8)$ & $2(2.1)$ \\
\hline Cannaboid/cannabinoid & $2(0.4)$ & $0(0.0)$ & I (2.9) & $0(0.0)$ & I (I.0) \\
\hline Headache/migraine medication & $32(6.6)$ & $8(3.4)$ & I (2.9) & $\mathrm{I}(0.9)$ & $22(22.9)$ \\
\hline Muscle relaxant & $69(14.3)$ & $47(19.8)$ & $6(17.1)$ & $13(1 \mathrm{I} .4)$ & $3(3.1)$ \\
\hline NSAID & $80(16.6)$ & $31(13.1)$ & $3(8.6)$ & $23(20.2)$ & $23(24.0)$ \\
\hline Opioid & $450(93.4)$ & $233(98.3)$ & $33(94.3)$ & $89(78.1)$ & $95(99.0)$ \\
\hline Steroid & $16(3.3)$ & $7(3.0)$ & $0(0.0)$ & $4(3.5)$ & $5(5.2)$ \\
\hline Tricyclic antidepressant & $35(7.3)$ & $5(2.1)$ & $\mathrm{I}(2.9)$ & $13(11.4)$ & $16(16.7)$ \\
\hline Other pain medication & $59(12.2)$ & $12(5.1)$ & $0(0.0)$ & $30(26.3)$ & $17(17.7)$ \\
\hline \multicolumn{6}{|c|}{ Most commonly used opioid medications (n, \%) } \\
\hline Codeine & $36(7.5)$ & $\mathrm{I}(0.4)$ & $2(5.7)$ & $0(0.0)$ & $33(34.4)$ \\
\hline Fentanyl & $42(8.7)$ & $15(6.3)$ & $8(22.9)$ & II (9.6) & $8(8.3)$ \\
\hline Hydrocodone or dihydrocodeine & $75(15.6)$ & $69(29.1)$ & $0(0.0)$ & $0(0.0)$ & $6(6.3)$ \\
\hline Hydromorphone & $31(6.4)$ & $6(2.5)$ & $3(8.6)$ & $22(19.3)$ & $0(0.0)$ \\
\hline Morphine & $76(15.8)$ & $36(15.2)$ & $4(I I .4)$ & $19(16.7)$ & $17(17.7)$ \\
\hline Oxycodone & $20 I(4 I .7)$ & $134(56.5)$ & $22(62.9)$ & $31(27.2)$ & $14(14.6)$ \\
\hline Tramadol & $72(14.9)$ & $19(8.0)$ & $3(8.6)$ & $5(4.4)$ & $45(46.9)$ \\
\hline Other opioid & $68(14.1)$ & $25(10.5)$ & $2(5.7)$ & $32(28.1)$ & $9(9.4)$ \\
\hline \multicolumn{6}{|l|}{ Comorbid conditions ( $\mathrm{n}, \%)$} \\
\hline Hypertension & I33 (27.6) & $62(26.2)$ & II (3I.4) & $34(29.8)$ & $26(27.1)$ \\
\hline Depression & $79(16.4)$ & $30(12.7)$ & $6(17.1)$ & $16(14.0)$ & $27(28.1)$ \\
\hline Anxiety & $56(11.6)$ & $31(13.1)$ & $7(20.0)$ & $4(3.5)$ & $14(14.6)$ \\
\hline Diabetes mellitus & $48(10.0)$ & $24(10.1)$ & $5(14.3)$ & $9(7.9)$ & $10(10.4)$ \\
\hline Hypothyroidism & $32(6.6)$ & II (4.6) & $4(11.4)$ & $7(6.1)$ & $10(10.4)$ \\
\hline Coronary artery disease & $18(3.7)$ & $5(2.1)$ & $3(8.6)$ & $6(5.3)$ & $4(4.2)$ \\
\hline
\end{tabular}

Notes: aledications for chronic pain documented in chart review from most recent treatment regimen; bopioid medications recorded in chart review with a prevalence of $5 \%$ or more.

Abbreviations: NSAID, non-steroidal anti-inflammatory drug; SNRI, serotonin-norepinephrine reuptake inhibitor.

Table 4 PAC-SYM items and subscales and additional at least moderate constipation symptoms

\begin{tabular}{|c|c|c|c|c|c|}
\hline & Overall $(n=493)$ & USA $(n=242)$ & Canada $(n=38)$ & Germany $(n=1 \mid 5)$ & UK $(n=98)$ \\
\hline Any symptom $\geq$ moderate present, $\mathrm{n}$ (\%) & $481(97.6)$ & $239(98.8)$ & $35(92.1)$ & $110(95.7)$ & $97(99.0)$ \\
\hline Discomfort in abdomen, $\mathrm{n}(\%)$ & $316(64.1)$ & $157(64.9)$ & $22(57.9)$ & $62(53.9)$ & $75(76.5)$ \\
\hline Pain in abdomen, $\mathrm{n}(\%)$ & $255(51.7)$ & $127(52.5)$ & $17(44.7)$ & 5 I (44.3) & $60(61.2)$ \\
\hline Bloating in abdomen, $\mathrm{n}(\%)$ & $338(68.6)$ & $150(62.0)$ & $23(60.5)$ & $83(72.2)$ & $82(83.7)$ \\
\hline Stomach cramps, n (\%) & $255(5 \mathrm{I} .7)$ & $140(57.9)$ & $22(57.9)$ & $34(29.6)$ & $59(60.2)$ \\
\hline Painful BM, n (\%) & $330(66.9)$ & I7I (70.7) & $23(60.5)$ & $62(53.9)$ & $74(75.5)$ \\
\hline Rectal burning during or after BM, $\mathrm{n}(\%)$ & $211(42.8)$ & $109(45.0)$ & $13(34.2)$ & $37(32.2)$ & $52(53.1)$ \\
\hline Rectal bleeding/tearing during or after BM, $\mathrm{n}(\%)$ & $|3|(26.6)$ & $6 I(25.2)$ & $6(15.8)$ & $31(27.0)$ & $33(33.7)$ \\
\hline Incomplete BM, n (\%) & $337(68.4)$ & $165(68.2)$ & $21(55.3)$ & $74(64.3)$ & $77(78.6)$ \\
\hline BMs too hard, n (\%) & $370(75.1)$ & $192(79.3)$ & $25(65.8)$ & $79(68.7)$ & $74(75.5)$ \\
\hline BMs too small, $n$ (\%) & $312(63.3)$ & $164(67.8)$ & $19(50.0)$ & $70(60.9)$ & $59(60.2)$ \\
\hline Straining/squeezing to pass BM, $\mathrm{n}(\%)$ & $407(82.6)$ & $202(83.5)$ & $28(73.7)$ & $89(77.4)$ & $88(89.8)$ \\
\hline Feeling like had to pass BM but could not, $n(\%)$ & $295(59.8)$ & $145(59.9)$ & $20(52.6)$ & $63(54.8)$ & $67(68.4)$ \\
\hline \multicolumn{6}{|l|}{ Additional symptoms } \\
\hline Nausea, n (\%) & $109(22.1)$ & $52(21.5)$ & $5(13.2)$ & $25(21.7)$ & $27(27.6)$ \\
\hline Vomiting, n (\%) & $28(5.7)$ & $16(6.6)$ & $2(5.3)$ & $4(3.5)$ & $6(6.1)$ \\
\hline Flatulence, n (\%) & $34 \mid(69.2)$ & $168(69.4)$ & $23(60.5)$ & $77(67.0)$ & $73(74.5)$ \\
\hline Gastroesophageal reflux, n (\%) & $181(36.7)$ & $106(43.8)$ & $15(39.5)$ & $28(24.3)$ & $32(32.7)$ \\
\hline Headache or migraine, $\mathrm{n}(\%)$ & $193(39.1)$ & $108(44.6)$ & $13(34.2)$ & $34(29.6)$ & $38(38.8)$ \\
\hline
\end{tabular}

Abbreviations: BM, bowel movement; PAC-SYM, Patient Assessment of Constipation-Symptoms. 
Table 5 Summary of natural/behavioral therapies and laxative utilization by category of therapy

\begin{tabular}{|c|c|c|c|c|c|}
\hline Use within each category (past 2 weeks) & Overall $(n=493)$ & USA $(n=242)$ & Canada $(n=38)$ & Germany $(n=\mid 15)$ & UK $(n=98)$ \\
\hline \multicolumn{6}{|l|}{ Natural/behavioral therapies, ${ }^{a} \mathrm{n}(\%)$} \\
\hline$\geq 1$ & $412(83.6)$ & $199(82.2)$ & $36(94.7)$ & $93(80.9)$ & $84(85.7)$ \\
\hline$\geq 2$ & $328(66.5)$ & 157 (64.9) & 27 (7I.I) & $76(66.1)$ & $68(69.4)$ \\
\hline$\geq 3$ & $191(38.7)$ & $91(37.6)$ & $14(36.8)$ & $50(43.5)$ & $36(36.7)$ \\
\hline \multicolumn{6}{|l|}{ OTC laxatives, ${ }^{\mathrm{b}} \mathrm{n}(\%)$} \\
\hline$\geq 1$ & $295(59.8)$ & $176(72.7)$ & $28(73.7)$ & $50(43.5)$ & $4 \mid(4 \mid .8)$ \\
\hline$\geq 2$ & $120(24.3)$ & $77(31.8)$ & $13(34.2)$ & $17(14.8)$ & $13(13.3)$ \\
\hline$\geq 3$ & $39(7.9)$ & $34(14.0)$ & $2(5.3)$ & $3(2.6)$ & $0(0.0)$ \\
\hline \multicolumn{6}{|l|}{ Prescription laxatives, ${ }^{c}$ n (\%) } \\
\hline$\geq 1$ & $91(18.5)$ & $19(7.9)$ & $3(7.9)$ & $51(44.3)$ & $18(18.4)$ \\
\hline$\geq 2$ & $7(1.4)$ & $3(1.2)$ & $0(0.0)$ & $\mathrm{I}(0.9)$ & $3(3.1)$ \\
\hline$\geq 3$ & $2(0.4)$ & $2(0.8)$ & $0(0.0)$ & $0(0.0)$ & $0(0.0)$ \\
\hline \multicolumn{6}{|c|}{ Natural/behavioral therapies and laxative combinations, $\mathrm{n}(\%)$} \\
\hline \multicolumn{6}{|l|}{ and $\geq$ I OTC only } \\
\hline$\geq$ I natural/behavioral therapies only & $|2|(24.5)$ & $52(21.5)$ & $9(23.7)$ & $22(19.1)$ & $38(38.8)$ \\
\hline$\geq$ I OTC only & $45(9.1)$ & $33(13.6)$ & I (2.6) & $7(6.1)$ & $4(4.1)$ \\
\hline$\geq \mathrm{I}$ natural/behavioral therapies, & $39(7.9)$ & $15(6.2)$ & $3(7.9)$ & $16(13.9)$ & $5(5.1)$ \\
\hline \multicolumn{6}{|l|}{ OTC, and $\geq I$ prescription laxative } \\
\hline$\geq \mathrm{I}$ natural/behavioral therapies & $44(8.9)$ & $4(1.7)$ & $0(0.0)$ & $30(26.1)$ & $10(10.2)$ \\
\hline \multicolumn{6}{|l|}{ and $\geq$ I prescription laxative only } \\
\hline$\geq$ I prescription laxatives only & $5(1.0)$ & $0(0.0)$ & $0(0.0)$ & $3(2.6)$ & $2(2.0)$ \\
\hline$\geq$ I prescription laxatives and OTC only & $3(0.6)$ & $0(0.0)$ & $0(0.0)$ & $2(1.7)$ & $\mathrm{I}(\mathrm{I} .0)$ \\
\hline No natural/behavioral therapy or laxative use & $28(5.7)$ & $10(4.1)$ & $\mathrm{I}(2.6)$ & $10(8.7)$ & $7(7.1)$ \\
\hline
\end{tabular}

Notes: ancludes probiotics, natural dietary changes, increased fluids, increased exercise, and fiber supplements; bincludes stool softeners, osmotic laxatives, saline laxatives, rectal options, and other OTC products; cincludes prescription osmotic laxatives, lactulose, Amitiza ${ }^{\circledR}$, Relistor ${ }^{\circledast}$, and other prescriptions.

Abbreviation: OTC, over-the-counter.

observational studies (ie, straining to pass a bowel movement, bowel movements too hard). ${ }^{4,10,31}$

Numerous other lower gastrointestinal symptoms were reported by $\geq 50 \%$ of patients in this study (eg, discomfort in abdomen, bloating in abdomen, painful bowel movements, incomplete bowel movements, wanting to pass a bowel movement but could not). Some of these commonly reported symptoms may also occur as side effects of conventional laxatives (eg, bloating, abdominal discomfort, flatulence). ${ }^{12}$
The prevalence of these symptoms suggests that patients may be undertreating their OIC and/or that the currently utilized therapies for the treatment of OIC may be lacking in efficacy and tolerability. Indeed, most patients in this study were either not using laxatives or were using them below clinically recommended frequencies or dosages. Among those who took sufficient doses of laxatives according to study criteria, an overwhelming $94 \%$ were found to have an inadequate response to them, defined as less than three bowel movements in the past 2 weeks and one or more

Table 6 Sufficiency of laxative use and inadequate response to laxatives

\begin{tabular}{|c|c|c|c|c|c|}
\hline & Overall $(n=493)$ & USA ( $n=242)$ & Canada $(n=38)$ & Germany ( $n=\mid 15)$ & UK $(n=98)$ \\
\hline Sufficient laxative use $\mathrm{a}^{\mathrm{a}}$ & $198(40.2)$ & $88(36.4)$ & $19(50.0)$ & $58(50.4)$ & $33(33.7)$ \\
\hline Insufficient laxative use ${ }^{b}$ & 118 (23.9) & $68(28.1)$ & $8(2 \mid . I)$ & $24(20.9)$ & $18(18.4)$ \\
\hline Nonlaxative use ${ }^{c}$ & 177 (35.9) & $86(35.5)$ & II (28.9) & $33(28.7)$ & $47(48.0)$ \\
\hline \multicolumn{6}{|l|}{ LIR status last 2 weeks } \\
\hline I $\times$ LIR status ${ }^{d}$ & $186(93.9)$ & $86(97.7)$ & $16(84.2)$ & $52(89.7)$ & $32(97.0)$ \\
\hline $2 \times$ LIR status $^{e}$ & $54(27.3)$ & $28(31.8)$ & $8(42.1)$ & $13(22.4)$ & $5(15.2)$ \\
\hline
\end{tabular}

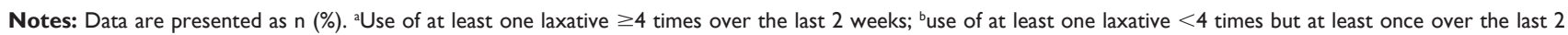
weeks; ' no reported laxative use over the last 2 weeks; ' 'sufficient laxative use (use of at least one laxative agent from a class $\geq 4$ times in the last 2 weeks) and inadequate response, defined as $<3$ BMs AND $\geq$ I PAC-SYM scored moderate, severe, or very severe; esufficient use of at least two laxative agents from at least two different classes $\geq 4$ times each in the last 2 weeks and inadequate response, defined as $<3$ BMs AND $\geq 1$ PAC-SYM scored moderate, severe, or very severe.

Abbreviations: BMs, bowel movements; LIR, inadequate response to laxatives; I $\times$ LIR, inadequate response to one laxative agent; $2 \times L I R$, inadequate response to $\geq 2$ agents from $\geq 2$ different laxative classes; PAC-SYM, Patient Assessment of Constipation-Symptoms. 
Table 7 WPAI-Constipation and EQ-5D

\begin{tabular}{|c|c|c|c|c|c|}
\hline & Overall $(n=493)$ & USA $(n=242)$ & Canada $(n=38)$ & Germany $(n=1 \mid 5)$ & UK $(n=98)$ \\
\hline Are you currently employed? (n, \% yes) & $132(26.8 \%)$ & $8 \mathrm{II}(33.5 \%)$ & $10(26.3 \%)$ & $17(14.8 \%)$ & $24(24.5 \%)$ \\
\hline \multicolumn{6}{|l|}{ If yes: during the past 7 days ... } \\
\hline ... how many hours did you miss from work & $\mathrm{n}=132$ & $n=81$ & $n=10$ & $\mathrm{n}=17$ & $\mathrm{n}=24$ \\
\hline $\begin{array}{l}\text { because of problems associated with your } \\
\text { constipation? (mean } \pm S D \text { ) hours }\end{array}$ & $4.6(11.9)$ & $6.2(13.9)$ & $1.9(4.2)$ & $0.2(0.8)$ & $3.2(10.4)$ \\
\hline ... how many hours did you miss from work & $n=132$ & $n=81$ & $n=10$ & $\mathrm{n}=17$ & $\mathrm{n}=24$ \\
\hline because of any other reason? (mean $\pm S D$ ) hours & $3.0(8.4)$ & $1.6(5.3)$ & $4.5(12.6)$ & $2.9(8.3)$ & $7.0(12.9)$ \\
\hline ... how many hours did you actually work? & $n=132$ & $n=8 \mid$ & $n=10$ & $\mathrm{n}=17$ & $n=24$ \\
\hline (mean $\pm S D$ ) hours & $30.8(16.4)$ & $36.3(13.7)$ & $29.8(15.0)$ & $27.7(14.0)$ & $15.0(16.7)$ \\
\hline \multicolumn{6}{|l|}{ WPAI-Constipation outcomes (mean \%, SD) } \\
\hline Percent work time missed & $n=120$ & $\mathrm{n}=79$ & $n=9$ & $\mathrm{n}=16$ & $n=16$ \\
\hline$(\text { absenteeism) })^{\mathrm{a}}$ & $8.9(20.0)$ & $10.1(18.9)$ & $4.8(9.5)$ & $0.8(2.3)$ & I3.I (34.0) \\
\hline Percent impairment while working & $\mathrm{n}=117$ & $\mathrm{n}=78$ & $n=9$ & $\mathrm{n}=16$ & $n=14$ \\
\hline$\left(\right.$ presenteeism) ${ }^{\mathrm{b}}$ & $32.2(21.9)$ & $38.3(21.3)$ & $31.1(16.9)$ & I4.4 (14.6) & $19.3(19.0)$ \\
\hline \multirow[t]{2}{*}{ Percent impairment in overall work productivity ${ }^{c}$} & $\mathrm{n}=117$ & $\mathrm{n}=78$ & $n=9$ & $n=16$ & $n=14$ \\
\hline & $29.0(19.3)$ & $33.8(18.6)$ & $29.2(16.2)$ & I4.3 (14.6) & $19.0(18.5)$ \\
\hline \multirow[t]{2}{*}{ Percent daily activity impairment ${ }^{d}$} & $n=493$ & $\mathrm{n}=242$ & $n=38$ & $\mathrm{n}=115$ & $n=98$ \\
\hline & $38.4(24.4)$ & $43.3(22.9)$ & $30.8(27.8)$ & $34.1(26.6)$ & 34.5 (21.9) \\
\hline \multirow[t]{2}{*}{ EQ-5D Index } & $n=449$ & $\mathrm{n}=214$ & $\mathrm{n}=28$ & $\mathrm{n}=\mathrm{II} \mid$ & $\mathrm{n}=96$ \\
\hline & $0.49(0.29)$ & $0.54(0.23)$ & $0.61(0.18)$ & $0.55(0.31)$ & $0.27(0.33)$ \\
\hline \multirow[t]{2}{*}{ EQ-5D VAS ${ }^{f}$} & $n=446$ & $n=211$ & $n=26$ & $n=113$ & $n=96$ \\
\hline & $50.6(21.6)$ & $52.9(21.9)$ & $58.6(21.4)$ & $45.0(19.2)$ & $49.9(22.5)$ \\
\hline
\end{tabular}

Notes: aNumber of hours missed due to constipation/(number of hours missed due to constipation + number of hours missed due to other reasons); ${ }^{\text {bdegree constipation }}$ affected productivity while working as reported on a $0-10$ scale, where 0 was "no effect" and 10 was "completely prevented productivity"/I0; 'number of hours missed due to constipation/(hours missed due to constipation + hours actually worked) + [( I - hours missed due to constipation)/(hours missed due to constipation + hours actually worked) $\times$ (degree constipation affected productivity while working/l0)]; dpercent daily activity impairment refers to degree constipation affected regular activities as

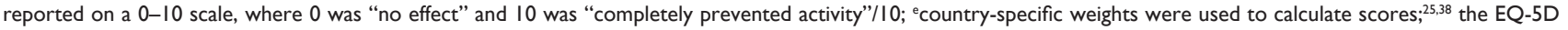
Index ranges from 0 (death) to I (full health); fthe EQ-5D VAS ranges from 0 (worst health state) to 100 (best imaginable health state).

Abbreviations: SD, standard deviation; EQ-5D, EuroQOL 5 Dimensions; VAS, visual analog scale; WPAI, Work Productivity and Activity Impairment.

constipation symptoms reported as moderate or greater over the same period.

Consistent with other literature, the present study also found that constipation symptoms interfered with opioid medication pain control, with almost half of patients (49\%) reporting moderate to complete interference with pain management resulting from their constipation. Eight percent of patients in the present study reported that they had changed how they took their opioid pain medications in order to have a bowel movement, which is a smaller proportion than that reported by Bell et al ${ }^{4}(35 \%)$ and Cook et al ${ }^{10}(27 \%)$. This discrepancy may be accounted for by the difference in recall periods used for this question across studies. In order to minimize recall bias, the present study asked about the past 7 days, while Cook et a ${ }^{10}$ asked about the past 4 weeks, and the recall period used by Bell et $\mathrm{al}^{4}$ was not specified.

The functional impact of the highly prevalent constipation symptoms and inadequate response to laxatives documented in this study was reflected in outcomes on the EQ-5D and WPAI-Constipation. The mean EQ-5D index score found in this sample (0.49) was somewhat lower than the mean EQ-5D index scores reported by Thomas et $\mathrm{al}^{28}$ in a sample of patients with chronic back pain (0.53) and those reported by Brazier et $\mathrm{al}^{27}$ in samples of patients with lower back pain (0.64) and IBS (0.66). In fact, of the seven patient populations evaluated by Brazier et al, ${ }^{27}$ only one group, ie, patients with osteoporosis recruited from a knee replacement waiting list or rheumatology clinic, scored similarly low (0.44). Patients with chronic obstructive pulmonary disease or leg ulcers, postmenopausal women, and elderly women all had higher mean scores (mean EQ-5D index range 0.54-0.73). Our results support the findings reported by Penning-van Beest et $\mathrm{al}^{5}$ in a sample of patients treated with opioids categorized as having nonadvanced illness (eg, chronic back pain, osteoarthritis) or advanced illness (eg, cancer) and stratified by presence of constipation to show the incremental impact of OIC on health-related quality of life. In that study, the median EQ-5D index was presented, and the results were considerably lower for patients with constipation regardless of type of chronic pain: 0.31 (nonadvanced illness and constipation) and 0.41 (advanced illness and constipation) compared with 0.65 
Table 8 Benefit, satisfaction, and willingness to continue treatment

\begin{tabular}{|c|c|c|c|c|c|}
\hline & Overall $(n=493)$ & USA $(n=242)$ & Canada $(n=38)$ & Germany $(n=\mid 15)$ & UK $(n=98)$ \\
\hline \multicolumn{6}{|c|}{ Have you had any benefit from your constipation treatment? n (\%) } \\
\hline No & $164(33.3 \%)$ & $79(32.6 \%)$ & $10(26.3 \%)$ & 39 (33.9\%) & $36(36.7 \%)$ \\
\hline Yes & $329(66.7 \%)$ & $163(67.4 \%)$ & $28(73.7 \%)$ & $76(66.1 \%)$ & $62(63.3 \%)$ \\
\hline \multicolumn{6}{|l|}{ If yes, how much benefit? n (\%) } \\
\hline Little benefit & $185(56.2 \%)$ & $112(68.7 \%)$ & $15(53.6 \%)$ & $29(38.2 \%)$ & $29(46.8 \%)$ \\
\hline Much benefit & $144(43.8 \%)$ & $51(31.3 \%)$ & $13(46.4 \%)$ & 47 (6I.8\%) & $33(53.2 \%)$ \\
\hline Taking all things into account, are you satisfied & $n=331$ & $\mathrm{n}=99$ & $n=23$ & $\mathrm{n}=\mathrm{II}$ & $\mathrm{n}=98$ \\
\hline \multicolumn{6}{|l|}{ with your constipation treatment? $\mathrm{n}(\%)^{\mathrm{a}}$} \\
\hline No & $184(55.6 \%)$ & $78(78.8 \%)$ & $12(52.2 \%)$ & $42(37.8 \%)$ & $52(53.1 \%)$ \\
\hline Yes & 147 (44.4\%) & $21(21.2 \%)$ & II (47.8\%) & 69 (62.2\%) & 46 (46.9\%) \\
\hline \multicolumn{6}{|l|}{ If yes, how satisfied? n (\%) } \\
\hline A little satisfied & 74 (50.3\%) & 17 (8I.0\%) & 7 (63.6\%) & $29(42.0 \%)$ & 21 (45.7\%) \\
\hline Very satisfied & 73 (49.7\%) & $4(19.0 \%)$ & $4(36.4 \%)$ & $40(58.0 \%)$ & 25 (54.3\%) \\
\hline \multicolumn{6}{|l|}{ If no, how dissatisfied? $\mathrm{n}(\%)$} \\
\hline A little dissatisfied & 89 (48.4\%) & 32 (4I.0\%) & $7(58.3 \%)$ & 27 (64.3\%) & $23(44.2 \%)$ \\
\hline Very dissatisfied & $95(51.6 \%)$ & $46(59.0 \%)$ & $5(41.7 \%)$ & $15(35.7 \%)$ & 29 (55.8\%) \\
\hline \multicolumn{6}{|c|}{ Would you be willing to continue constipation treatment with this medication? n (\%) } \\
\hline No & 31 (6.3\%) & $24(9.9 \%)$ & $3(7.9 \%)$ & $2(1.7 \%)$ & $2(2.0 \%)$ \\
\hline Yes & 462 (93.7\%) & $218(90.1 \%)$ & 35 (92.1\%) & $113(98.3 \%)$ & 96 (98.0\%) \\
\hline \multicolumn{6}{|l|}{ If yes, how willing? n (\%) } \\
\hline A little bit willing & $125(27.1 \%)$ & 77 (35.3\%) & $10(28.6 \%)$ & $12(10.6 \%)$ & $26(27.1 \%)$ \\
\hline Very willing & 334 (72.3\%) & $139(63.8 \%)$ & $25(71.4 \%)$ & 100 (88.5\%) & $70(72.9 \%)$ \\
\hline Missing & $3(0.6 \%)$ & $2(0.9 \%)$ & $0(0.0 \%)$ & I (0.9\%) & $0(0.0 \%)$ \\
\hline \multicolumn{6}{|l|}{ If no, how unwilling? n (\%) } \\
\hline A little unwilling & $22(71.0 \%)$ & 17 (70.8\%) & $3(100.0 \%)$ & $2(100.0 \%)$ & $0(0.0 \%)$ \\
\hline Very unwilling & $9(29.0 \%)$ & $7(29.2 \%)$ & $0(0.0 \%)$ & $0(0.0 \%)$ & $2(100.0 \%)$ \\
\hline
\end{tabular}

Note: a Due to a problem with the programming of the survey, this question was not presented to 162 participants and data are missing.

(nonadvanced illness and no constipation) and 0.61 (advanced illness and no constipation). The median EQ-5D index in the present study was 0.51 , higher than that found in either group with constipation in the Penning-van Beest study, ${ }^{5}$ but lower than that found in patients with no constipation. In parallel, the mean EQ-5D VAS (50.6) in the present study was similar to findings in patients with constipation with advanced illness (49.5) and nonadvanced illness (51.7) as well as those with no constipation in the advanced illness group (50.4). Patients with nonadvanced illness and no constipation in that study scored considerably higher $(0.65)$.

Among those who were working in this study, the overall work productivity loss (32\%) is the equivalent of 14 hours of lost productivity per week. These results are consistent with data from a psychometric validation study establishing the WPAI-SHP for use in patients with IBS, a similarly impactful bowel disorder (absenteeism 9\% [present study], 4\% ${ }^{22}$; presenteeism 32\% [present study], 32\% $\%^{22}$; overall work productivity loss $29 \%$ [present study], 34\% $\%^{22}$; impairment in daily activities $38 \%$ [present study], $41 \%{ }^{22}$ ). Supporting this, other research using the WPAI-General Health tool has found higher levels of absenteeism and lower levels of presenteeism, diminished overall work productivity, and impairment in activities for patients with OIC as compared with healthy controls ${ }^{3}$ and for patients with IBS with constipation (as compared with those who did not have IBS with constipation). ${ }^{32}$

In parallel with the considerable health-related quality of life burden of OIC and lack of efficacious treatments documented in this study, less than half of our participants (44\%) reported being satisfied with their therapy for OIC, similar to the rate of overall satisfaction found in prior research. ${ }^{12}$ Perhaps most striking was the finding that almost all patients reported a willingness to continue the constipation treatment that they were currently using. As the findings for inadequate response to laxatives revealed, for the majority $(60 \%)$ of patients, treatment for constipation either did not include any laxatives or included infrequent doses. Furthermore, nearly all those who took sufficient doses of laxatives had an inadequate response.

The fact that many of these patients reported a willingness to continue this treatment approach despite persistent constipation with substantial impacts on their activities of daily living and work productivity is puzzling. These seemingly inconsistent findings may reflect a resignation to 
longstanding symptoms of constipation. Reflective of the chronic noncancer pain patient population, most participants in this study had long histories of enduring chronic pain and being treated with opioids (mean duration of opioid use, 6.4 years). As such, many may have "given up" on laxatives, either due to tolerability problems, limited efficacy, or some combination of these. Patients may also be unaware of recent advances in therapeutic approaches for OIC to manage and prevent $\mathrm{OIC}$ without compromising analgesic efficacy. ${ }^{33}$ Consistent with this, over a third (37\%) of patients in this study did not discuss constipation with their health care provider at their most recent office visit. Data from a recent systematic review and meta-analysis support the use of $\mu$-opioid receptor antagonists in the treatment of OIC, and suggest a need for national or international guidelines to aid gastroenterologists and other clinicians in identifying appropriate therapies for OIC. ${ }^{34}$

The present study has a number of important strengths and limitations that require elucidation. ${ }^{35}$ First, an important strength is the rigorous study methodology. The use of clinic-based patient recruitment provided medical verification of opioid dosage and additional information about pain and concomitant medication use and comorbid conditions. Prior observational studies focusing on noncancer pain have relied exclusively on patient-reported data and have recruited participants from consumer panels constructed by online market research providers. ${ }^{4,10}$ In contrast to Bell et al, ${ }^{4}$ the methodology in the present study did not stipulate that participants be currently taking laxatives, a distinction that sheds light on the prevalence and patterns of laxative utilization in this patient population by exposing a substantial proportion of nonlaxative users. Despite these methodological differences, the demographic and clinical characteristics reported in these prior studies are extremely similar to those found in the present study with respect to sex, type of chronic pain, and pain severity.

The use of condition-specific patient-reported outcome instruments supported by evidence of reliability and validity in relevant patient populations is a second strength of the present study. Short recall periods (eg, 24 hours, 7 days, 2 weeks) were used whenever possible in order to minimize recall bias. A qualification to this overall strength is the BSW which, although relevant to this study population, was developed and psychometrically evaluated for use in overactive bladder and, to our knowledge, has not been used to assess satisfaction with therapies for constipation. Therefore, more research is needed to evaluate its content validity and psychometric performance in patients with OIC.
A limitation to the findings presented here is that it is impossible to determine the influence of any one treatment or medication on outcomes due to utilization of polypharmacy and considerable comorbidity found in this real world sample of patients with chronic pain and OIC. Although post hoc analyses conducted to explore outcomes by laxative class did not show any statistically significant differences, longitudinal data and data from controlled study designs are needed to further disentangle the impact of specific therapies and conditions on patient outcomes.

A second limitation concerns the potential for bias resulting from patient report and the use of an Internetbased survey conducted in multiple countries. Data on natural/behavioral therapy and laxative utilization are based exclusively on patient report and may be subject to inaccuracy or recall bias, despite efforts to provide participants with pictures of different products in order to assist them in responding to these questions. The use of an Internetbased platform for survey administration may have biased the sample toward participants having a greater familiarity with the Internet and higher socioeconomic status and educational attainment. ${ }^{36}$ This limitation notwithstanding, a study in patients with inflammatory bowel disease found good accuracy for self-reported medical information via Internet-based research when patients were engaged with the clinical site. ${ }^{37}$

\section{Conclusion}

Taken together, these findings suggest that patients treated with opioids for chronic noncancer pain commonly endure symptoms of constipation that limit their activities of daily living, work productivity, overall health-related quality of life, and, in some cases, their ability to manage their pain effectively, while adhering to approaches to treat OIC that provide little relief. The mean EQ-5D index score found in this study indicates a lower level of health-related quality of life than that found in samples of patients with chronic pain alone or other chronic medical conditions. Longitudinal data in this ongoing study will be important to better understand how participants manage their pain and OIC over time. Patients newly treated with opioids may cycle through numerous therapies for constipation with little benefit before resigning themselves to their symptoms and therapeutic nihilism. Thus, more research is needed to identify more efficacious constipation therapies for this patient population. Clinicians should be encouraged to proactively assess OIC. International guidelines are needed to help clinicians identify appropriate therapies for the treatment of OIC. 


\section{Acknowledgments}

The authors would like to acknowledge the work of the following employees of UBC: an Express Scripts Company who contributed to the study design and implementation and to the data collection: Krista A Payne, Colleen Valenzula, Jersino Jean-Mary, and Andrew Goldwin. The authors would also like to acknowledge the work of the following Evidera employees who contributed to the data analysis: Christine Thompson, Hilary Wilson, and Ning Wu. The authors would also like to acknowledge the work of the following AstraZeneca employees who contributed to the study design and implementation: Fred King, Soheil Chavoshi, Ron Dirienzi, Berhanu Alemayehu and Joe Crawley.

\section{Disclosure}

KSC and CCS are employees of Evidera and were paid scientific consultants to AstraZeneca in connection with the Burden of Illness of Opioid-induced Constipation study and manuscript. KY is an employee of UBC: an Express Scripts Company who was a paid scientific consultant to AstraZeneca in connection with the Burden of Illness of Opioid-induced Constipation study and manuscript. RJL and CJD are employees of AstraZeneca. JT is a physician and received financial support from AZ for his scientific consultation in designing and interpreting this study. The authors have no other conflicts of interest in this work.

\section{References}

1. Quigley C. Opioid switching to improve pain relief and drug tolerability. Cochrane Database Syst Rev. 2004;3:CD004847.

2. Goodheart CR, Leavitt SB. Managing opioid-induced constipation in ambulatory care patients. Pain Topics. 2006. Available from: http:// updates.pain-topics.org/pdf/Managing_Opioid-Induced_Constipation. pdf. Accessed March 18, 2014.

3. Leslie J, Bell T, Annunziata K, Freedman D. Opioid-induced constipation compromises pain management and impacts patient quality of life. Anesthesiology. 2006;105:Abstr 1490.

4. Bell TJ, Panchal SJ, Miaskowski C, et al. The prevalence, severity, and impact of opioid-induced bowel dysfunction: results of a US and European Patient Survey (PROBE 1). Pain Med. 2009;10(1): 35-42.

5. Penning-van Beest FJ, van den Haak P, Klok RM, Prevoo YF, van der Peet DL, Herings RM. Quality of life in relation to constipation among opioid users. J Med Econ. 2010;13(1):129-135.

6. Hjalte F, Berggren AC, Bergendahl H, Hjortsberg C. The direct and indirect costs of opioid-induced constipation. J Pain Symptom Manage. 2010;40(5):696-703.

7. Boswell K, Kwong WJ, Kavanagh S. Burden of opioid-associated gastrointestinal side effects from clinical and economic perspectives: a systematic literature review. J Opioid Manag. 2010;6(4):269-289.

8. Panchal SJ, Muller-Schwefe P, Wurzelmann JI. Opioid-induced bowel dysfunction: prevalence, pathophysiology and burden. Int J Clin Pract. 2007;61(7):1181-1187.

9. Brown RT, Zuelsdorff M, Fleming M. Adverse effects and cognitive function among primary care patients taking opioids for chronic nonmalignant pain. J Opioid Manag. 2006;2(3):137-146.
10. Cook SF, Lanza L, Zhou X, et al. Gastrointestinal side effects in chronic opioid users: results from a population-based survey. Aliment Pharmacol Ther. 2008;27(12):1224-1232.

11. Allan L, Hays H, Jensen NH, et al. Randomised crossover trial of transdermal fentanyl and sustained release oral morphine for treating chronic non-cancer pain. BMJ. 2001;322(7295):1154-1158.

12. Pappagallo M. Incidence, prevalence, and management of opioid bowel dysfunction. Am J Surg. 2001;182(Suppl 5A):11S-18S.

13. Candy B, Jones L, Goodman ML, Drake R, Tookman A. Laxatives or methylnaltrexone for the management of constipation in palliative care patients. Cochrane Database Syst Rev. 2011;1:CD003448.

14. Kelly P, Cook SF, Kaufman DW, et al. Prevalence and characteristics of opioid use in the US adult population. Pain. 2008;138(3): 507-513.

15. Frank L, Kleinman L, Farup C, Taylor L, Miner P Jr. Psychometric validation of a constipation symptom assessment questionnaire. Scand J Gastroenterol. 1999;34(9):870-877.

16. Lowenstein O, Leyendecker P, Hopp M, et al. Combined prolongedrelease oxycodone and naloxone improves bowel function in patients receiving opioids for moderate-to-severe non-malignant chronic pain: a randomised controlled trial. Expert Opin Pharmacother. 2009;10(4):531-543.

17. Michna E, Blonsky ER, Schulman S, etal. Subcutaneous methylnaltrexone for treatment of opioid-induced constipation in patients with chronic, nonmalignant pain: a randomized controlled study. J Pain. 2011;12(5):554-562.

18. Simpson K, Leyendecker P, Hopp M, et al. Fixed-ratio combination oxycodone/naloxone compared with oxycodone alone for the relief of opioid-induced constipation in moderate-to-severe noncancer pain. Curr Med Res Opin. 2008;24(12):3503-3512.

19. Sloots CE, Rykx A, Cools M, Kerstens R, De Pauw M. Efficacy and safety of prucalopride in patients with chronic noncancer pain suffering from opioid-induced constipation. Dig Dis Sci. 2010;55(10):2912-2921.

20. Meissner W, Leyendecker P, Mueller-Lissner S, et al. A randomised controlled trial with prolonged-release oral oxycodone and naloxone to prevent and reverse opioid-induced constipation. Eur J Pain. 2009;13(1):56-64.

21. Reilly MC, Zbrozek AS, Dukes EM. The validity and reproducibility of a work productivity and activity impairment instrument. Pharmacoeconomics. 1993;4(5):353-365.

22. Reilly MC, Bracco A, Ricci JF, Santoro J, Stevens T. The validity and accuracy of the Work Productivity and Activity Impairment questionnaire - irritable bowel syndrome version (WPAI: IBS). Aliment Pharmacol Ther. 2004;20(4):459-467.

23. Bushnell DM, Reilly MC, Galani C, et al. Validation of electronic data capture of the Irritable Bowel Syndrome - Quality of Life Measure, the Work Productivity and Activity Impairment Questionnaire for Irritable Bowel Syndrome and the EuroQol. Value Health. M 2006;9(2):98-105.

24. Reilly MC, McBurney CR. Responsiveness of the Work Productivity and Activity Impairment Questionnaire for Irritable Bowel Syndrome with Constipation (WPAI: IBS-C) to clinically meaningful change. Poster presented at the annual meeting of the American College of Gastroenterology, October 30 to November 2, 2005, Honolulu, HI, USA.

25. Rabin R, Oemar M, Oppe M. EQ-5D-3L User Guide: Basic Information on How to Use the EQ-5D-3L Instrument. Rotterdam, The Netherlands: The EuroQol Group; 2011.

26. Bushnell DM, Martin ML, Ricci JF, Bracco A. Performance of the EQ-5D in patients with irritable bowel syndrome. Value Health. 2006;9(2):90-97.

27. Brazier J, Roberts J, Tsuchiya A, Busschbach J. A comparison of the EQ-5D and SF-6D across seven patient groups. Health Econ. 2004;13(9):873-884.

28. Thomas KJ, MacPherson H, Ratcliffe J, et al. Longer term clinical and economic benefits of offering acupuncture care to patients with chronic low back pain. Health Technol Assess. 2005;9(32):iii-iv, ix-x, 1-109. 
29. Pleil AM, Coyne KS, Reese PR, et al. The validation of patient-rated global assessments of treatment benefit, satisfaction, and willingness to continue - the BSW. Value Health. 2005;8 Suppl 1:S25-S34.

30. Santos JC, Telo ER. Solifenacin: scientific evidence in the treatment of overactive bladder. Arch Esp Urol. 2010;63(3):197-213.

31. Tuteja AK, Biskupiak J, Stoddard GJ, Lipman AG. Opioid-induced bowel disorders and narcotic bowel syndrome in patients with chronic non-cancer pain. Neurogastroenterol Motil. 2010;22(4):424-430, e96.

32. Bracco A, Reilly MC, McBurney CR, Ambegaonkar B. Burden of irritable bowel syndrome with constipation on healthcare resource utilization, work productivity, activity impairment and quality of life in France, Germany and the United Kingdom. Paper presented at the 13th United European Gastroenterology Week, October 15-20, 2005, Copenhagen, Denmark.

33. Reimer K, Hopp M, Zenz M, et al. Meeting the challenges of opioidinduced constipation in chronic pain management - a novel approach. Pharmacology. 2009;83(1):10-17.
34. Ford AC, Brenner DM, Schoenfeld PS. Efficacy of pharmacological therapies for the treatment of opioid-induced constipation: systematic review and meta-analysis. Am J Gastroenterol. 2013;108(10):1566-1574.

35. Puhan MA, Akl EA, Bryant D, et al. Discussing study limitations in reports of biomedical studies- the need for more transparency. Health Qual Life Outcomes. 2012;10:23.

36. Bhinder S, Chowdhury N, Granton J, et al. Feasibility of internet-based health-related quality of life data collection in a large patient cohort. J Med Internet Res. 2010;12(3):e35.

37. Kelstrup AM, Juillerat P, Korzenik J. The accuracy of self-reported medical history: a preliminary analysis of the promise of Internet-based research in inflammatory bowel diseases. J Crohns Colitis. October 30, 2013. [Epub ahead of print.]

38. Bansback N, Tsuchiya A, Brazier J, Anis A. Canadian valuation of EQ-5D health states: preliminary value set and considerations for future valuation studies. PLoS One. 2012;7(2):e31115.
ClinicoEconomics and Outcomes Research

\section{Publish your work in this journal}

ClinicoEconomics \& Outcomes Research is an international, peerreviewed open-access journal focusing on Health Technology Assessment, Pharmacoeconomics and Outcomes Research in the areas of diagnosis, medical devices, and clinical, surgical and pharmacological intervention. The economic impact of health policy and health systems

\section{Dovepress}

organization also constitute important areas of coverage. The manuscript management system is completely online and includes a very quick and fair peer-review system, which is all easy to use. Visit http://www.dovepress.com/testimonials.php to read real quotes from published authors.

Submit your manuscript here: http://www.dovepress.com/clinicoeconomics-and-outcomes-research-journal 